\title{
Effect of infant stimulation on the adaptation to birth: a randomized trial*
}

\author{
Lucy Marcela Vesga Gualdrón ${ }^{1}$ \\ (1) https://orcid.org/0000-0003-1575-1782 \\ María Mercedes Durán de Villalobos ${ }^{1}$ \\ (iD) https://orcid.org/0000-0003-3431-1264
}

* Paper extracted from doctoral dissertation "Result of auditory, tactile, visual and vestibular stimulation (ATVV) in the adaptation to the birth of the mother and child dyad", presented to Universidad Nacional de Colombia, Facultad de Enfermería, Bogotá, Colombia.

1 Universidad Nacional de Colombia, Facultad de Enfermería, Sede Bogotá D.C, CO, Colombia.
Objective: to measure the effect of an infant stimulation therapy (auditory, tactile, visual and vestibular) on the adaptation to postnatal life of the mother-child dyad. Method: an experimental and blind study composed of 120 dyads of first-time mothers and full-term newborns, who practiced breastfeeding. The follow-up was conducted during the first five weeks of life and the evaluation was carried at two different times. Results: the adaptive capacity was measured in two modes. The physiological adaptive mode (activity and exercise and neonatal nutrition) and the interdependence adaptive mode (appropriate affection and proper development); and statistically significant differences were found in favor of the experimental group. Regression models that show the collaborative relationship between mother and child, and their reciprocity in the process of adaptation were proposed. Conclusion: the early stimulation is a therapy with bidirectional effect, because it has favorable effects on the person who administers it; promotes health and prevents illness in the process of adaptation to birth; especially in contexts of vulnerability. It is recommended its teaching to mothers and its application in the home environment. This study was registered in the Australian New Zealand Clinical Trial Registry (ANZCTR) under protocol number: ACTRN12617000449336.

Descriptors: Postnatal Care; Adaptation; Physical Stimulation; Health Promotion; Maternal and Child Health; Postpartum Period.

\section{Como citar este artigo}

Gualdrón LMV, Villalobos MMD. Effect of infant stimulation on the adaptation to birth: a randomized trial. Rev. Latino-Am. Enfermagem. 2019;27:e3176. [Access DOI: http://dx.doi.org/10.1590/1518-8345.2896.3176.

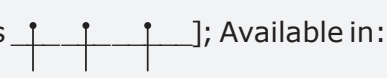

month day year

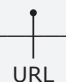




\section{Introduction}

During pregnancy, the mother-child dyad begins, which is united through a symbiotic relationship in which the fetus takes from its mother what is necessary to grow and develop. After birth, the dyad changes its way of relating. This significant moment in the life of human beings is full of changes, which transform their way of interacting(1). Mother and child face the challenge of understanding each other, and their relationship must be synchronous, since his well-being depends on her(2). However, neither the mothers nor the newborns have the best time during the first days of postnatal life. For most people, motherhood is a wonderful experience; however, physiological, emotional and behavioral adjustments are experienced during the course of the adaptation process. The physiological adjustments in women show that a poor health status is associated with the mode of birth. This was the conclusion of a systematic literature review that included studies from 1996 to 2014. Sixty-two studies were selected because of their high methodological quality, which allow it to be stated that postpartum mothers have pain (headache, breast pain, backache, abdominal pain, pain after episiorrhaphy or hysterorrhaphy); fatigue and a low level of energy due to the scarce hours of rest; and they may present urinary incontinence. Thus, due to all the above factors, they have a diminished physical quality of life $\mathrm{e}^{(3)}$. The newborn tests the functioning of its organs and systems, faces the challenge of feeding himself and maintaining the metabolic and hydration balance. This is a fundamental skill that will be further improved as the days go by, but if the initial difficulty is maintained, its health and growth may be adversely impacted ${ }^{(4-6)}$. Emotional adjustments in the newborn are manifested with a high level of stress $^{(1)}$. A systematic review that included 15 studies of very good methodological quality evaluated the physiological and behavioral signs that newborns have in response to nociceptive stimuli associated with stress ${ }^{(7)}$. This review concluded that the responses are associated with the gestational age and are fundamentally changes in the heart rate and oxygen saturation, facial expressions, such as frowning, squeezing the eyes shut and marking the nasolabial sulcus, crying, among other signs ${ }^{(8)}$. The mother experiences a high level of anxiety, stress related to the new role, emotional sensitivity and depressive symptoms ${ }^{(9-10)}$. Behavioral adjustments are characterized by a lack of maternal skills, which increases when it is first-time mother or when the delivery is performed through a cesarean section $^{(11)}$. In turn, the newborn does not know many of the environmental stimuli, so during the first days of life it has difficulty to maintain a waking state that allows it to interact with its environment, and it is not able to maintain a state of calm alertness easily, what makes the process of feeding it even more difficult ${ }^{(12)}$. The greater the difficulty of the dyad in adapting, the greater the risk of postpartum depression (PPD) ${ }^{(3,10)}$. PPD makes the mother-child interaction difficult and causes delays in child growth and development ${ }^{(3,10-11,13)}$.

Therefore, support strategies such as home visits are of great importance. These strategies are provided in different health systems with the aim of preventing habitual complications and hospital readmissions. However, developing countries such as Colombia do not provide comprehensive coverage and follow-up during this process, which is considered a highly sensitive period, and in which there is an increased risk of illness or death of the mother-child dyad ${ }^{(14)}$. These findings worry the nursing professionals, as it requires low-cost strategies that promote health and prevent diseases in this important period. Different studies show the positive effects of the early auditory, tactile, visual and vestibular stimulation therapy (ATVV), mainly in hospitalized premature newborns and their families ${ }^{(15-17)}$. In addition, it is presented as potentially effective when applied in the home context to benefit the process of adapting to postnatal life, since it allows the organization of the newborn behavior, which is expressed as the effective sucking ability, and that requires the hierarchical integration of social interaction systems: sensorial, autonomous, motor and behavioral(15). It promotes growth $^{(16-17)}$ and the emotional adjustments of the mother ${ }^{(17)}$. In this way, it is expected that the beneficial effects promote a favorable adaptation of the physiological adaptive mode (nutrition and activity and rest) and the interdependence adaptive mode (appropriate affection and proper development) of the mother-child dyads. If the newborn organizes its behavior sooner, its signals will be clearer and the relationship with its mother will be more synchronous. The effective sucking allows longer periods of neonatal sleep, contributing to the mother's rest and the prevention of emotional problems ${ }^{(18)}$. The objective of this study was to measure the effect of the early stimulation therapy ATVV on the adaptation to postnatal life of the mother-child dyad. The adaptation was measured through the physiological adaptive (nutrition and activity and rest) and interdependence adaptive modes (appropriate affection and proper development).

\section{Method}

Experimental study of equivalent materials determined by the mode of birth with one-to-one selection ratio, since the vaginal delivery or caesarean section can 
affect the outcome variables in a particular way, and repeated measurements were performed. In total, 120 dyads were approached, in the collective accommodation service of a tertiary-level hospital institution located in the city of Bogotá D.C., Colombia, from the first 24 hours after birth until the 5 weeks of postnatal life. The following criteria were considered: being first-time mothers with healthy term newborns (Apgar higher than or equal to 8 at 5 minutes) and being able to read and write. As exclusion criteria: mothers aged under 18 years, with psychological or learning disorders with previous medical diagnosis; newborns weighing less than 2,500 grams and dyads who required hospitalization during follow-up or who had stopped breastfeeding. The experimental group received the standard care of the institution and was instructed on the multisensory stimulation therapy ATVV for its home application 2 times a day, during the first two weeks. The first author trained the mothers to perform auditory stimulation with her own voice; tactile stimulation by means of sequential massage; visual stimulation by means of constant visual contact, and vestibular stimulation, cradling in their arms. This therapy follows the protocol described by American researchers in 1994, and evaluates the newborn responses of liking or disliking, which allows to adjust the intensity of the stimuli(19). The training of the mothers followed a protocol supported by the use of audiovisual tools designed specifically for this study, which ensured the standardization of the teaching of this technique. The mothers were approached in the best possible comfort conditions and the training was offered after watching a video that described how to perform the steps of this therapy. Subsequently, the mothers were given headphones so they could perform the therapy by listening to the step-by-step instructions. The training was evaluated using a checklist. To perform the therapy at home, an audio recording was provided with the step-bystep instructions that controlled the duration and order of application of the stimuli, and ensured the reproducibility of the therapy under optimal quality conditions. The control group received the standard care of the institution and once the study follow-up was completed, this group was trained on the technique of early stimulation as a way of ethical compensation. To measure the adaptation to postnatal life, the research was guided by the Roy Adaptation Model $\left(\right.$ RAM) ${ }^{(20)}$, who states that the process can take place in Adaptive Modes. This study focused on the physiological adaptive mode (nutrition and activity and rest) and the interdependence adaptive mode (appropriate affection and proper development). Physiological Adaptive Mode: Through physical activity, people carry on with their daily life, relate to the environment and other beings; while rest provides moments of relaxation and repose so that the body can restore the energies. The balance between activity and sleep depends on the balance between these two processes. According to Roy's model, situations such as pain, psychological stress and sleep disturbances affect the balance between activity and rest. Nutrition is the process by which an individual takes and assimilates the necessary food to maintain its human functioning, promote its growth and repair tissue damage or injuries. In this need, two basic processes are described, which are the digestive process and the metabolic process ${ }^{(20)}$. Interdependence Adaptive Mode: Appropriate affection includes the willingness and ability to give and receive love, respect, courage, education, knowledge, ability, time, loyalty. It includes the need to be nurtured in terms of care, attention, acceptance and understanding. Proper development refers to the process associated with learning and maturity. The appropriate balance between these factors influences the ability to adapt and achieve relational integrity (20). Figure 1 shows the articulation between the concepts or outcome variables and the instruments used in the measurement in this study. All the instruments used were tested for their validity and reliability.

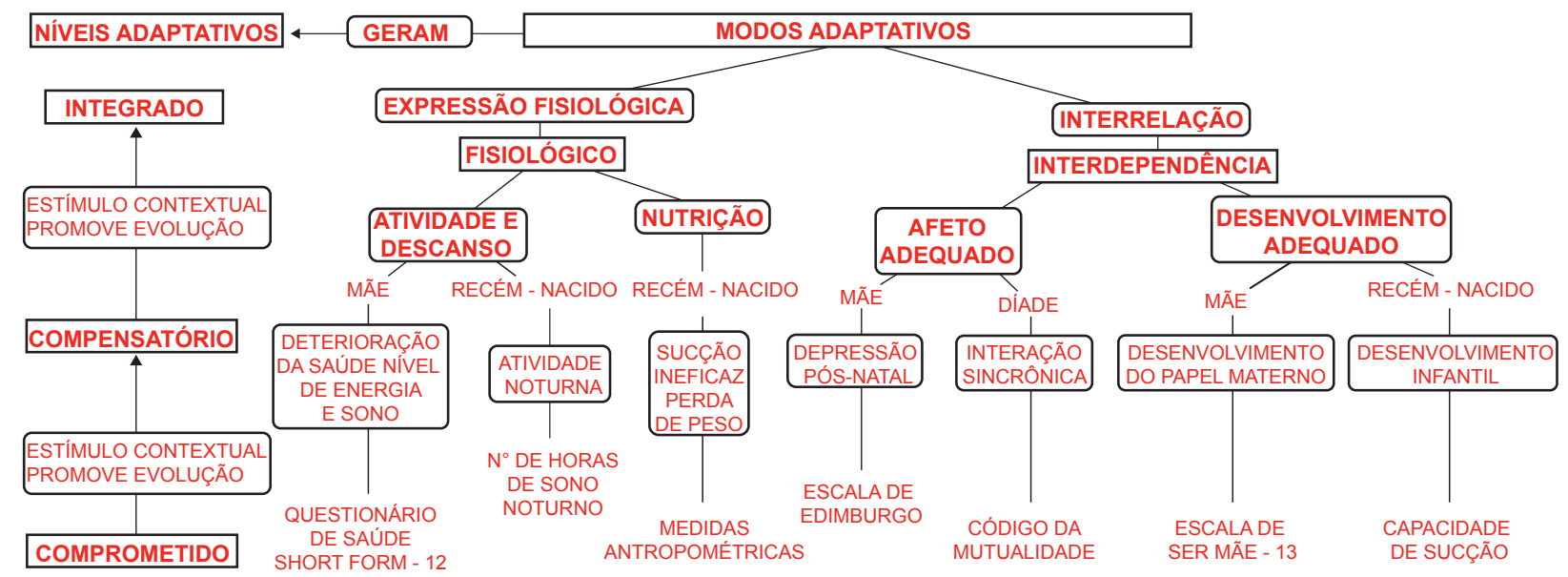

Figure 1 - Roy's Adaptation Model and Outcome Variables. Bogotá D.C, Colombia, 2016 
The mother-child dyads were observed in two moments, during the $2^{\text {nd }}$ and $5^{\text {th }}$ weeks of postnatal life. The newborns were cited 2 hours after the last intake, so that they had their sucking observed. Initially, a pediatrician performed the anthropometric measurements (weight, height and cephalic perimeter) and the mother was instructed to breastfeed her newborn. The procedure was video recorded from beginning to end. Once breastfeeding ended, a research assistant weighed the newborn again. The difference calculated in grams was attributed to the amount of milk ingested, in cubic centimeters, to determine the sucking ability.

Two specialists evaluated the video recordings to measure the effective sucking time and the number of suctions and saved them. The first minutes allowed the analysis of the Dyadic Mutuality Code. The mothers completed the questionnaires for self-reporting. The sample size was calculated by the following mathematical expression:

$$
n=2\left\{\sqrt{x_{1-\alpha}^{2}(k-1)-(k-2)+Z_{1-\beta}}\right\}^{2}\left(\frac{\sigma}{\Delta}\right)^{2}+1
$$

Where:

a: probability of committing a type I error $=(0.04)$ $\beta$ : probability of committing type II error $=(0.01) \sigma$ : upper bound of the standard deviation using the values produced by the variable total score of the scale used $\Delta$ : Minimum difference between the averages of the groups to calculate the $\beta$ value $=(0.8 \sigma) \mathrm{k}$ : number of groups to be compared $=(2)$. A sample composed of 60 dyads was calculated for each experimental and control group.

For the random assignment in the intervention, the authors created two blocks of 50 different random numbers between 1 and 100, using Excel's RAND function. A block of random numbers was assigned to births by vaginal delivery and the other block to cesarean delivery births. The participants were consecutively assigned and, according to this sequence, assigned to each experimental group (EG) or control group (CG) using the corresponding table. Therefore, the same number of births by vaginal delivery and cesarean delivery births was assigned in each group. The professionals who participated as evaluators did not know the groups assignment. Mothers were informed of the beneficial effects of the early stimulation therapy on child development, but they were not informed of the effects on growth and maternal benefits.
Data analysis was carried out using the statistical package SPSS version 22. All tests were performed with a $95 \%$ confidence interval and two main stages were followed: one to estimate the differences between the experimental and control groups using the Mann-Whitney $U$ test; and the other one to estimate the differences between each group at the two assessment moments using the Wilcoxon test, considering a non-normal distribution of the data. Regression models were proposed(21). Additionally, G*Power 3.1.9.2 free software was used to calculate the effect size (ES), estimated by the Cohen's D values using the differences between means. The research was approved by the ethics committee of the nursing faculty of the National University of Colombia and by the research ethics committee of the health institution where the study was conducted.

\section{Results}

The fieldwork was carried out from July 19 to November 27, 2016, at a Hospital in the city of Bogotá, D.C., Colombia. Taking into account the loss of participants, a larger number of participants was approached. However, despite the efforts undertaken, it was not possible to count on the estimated sample size at the end of the follow-up. Figure 2 shows the flow chart.

The greatest damages were observed in those mothers who underwent cesarean section, since mobility restriction in the days after delivery was a recurrent cause of their nonattendance at the evaluation meetings. Table 1 shows the description of the sociodemographic characteristics of participants and reveals that they are mothers with a low level of education, employed in low-skilled jobs, with a family income of around 320 dollars per month, that is, equivalent to a little more than one local minimum wage. The average length of labor was 8 hours.

The newborns exhibited a similar distribution, according to sex and APGAR scores. Other variables describing the physiological maturation of the newborns are shown in Table 2, which reveals in detail that the anthropometric measures and the gestational age determined by the pediatrician using the Ballard test at birth are similar between the groups.

The analyses show that the groups are homogeneous between themselves, an indispensable feature to evaluate the effect of the early stimulation therapy. Nonparametric tests were performed to 
evaluate the differences between the experimental and control groups at the two assessment moments. In the physiological adaptive mode: Activity and rest, the number of hours of night sleep of the mother and newborn was measured and no statistically significant difference was found by the Mann-Whitney $U$ test ( $p$ : 0.268 ) at the first measurement point. In contrast, statistically significant differences were found at the second measurement point ( $p$ : 0.034) CG (Mean: 7.82 hours, SD: 1.152 ) vs EG (Mean: 8.33 hours, SD: 1.194) (Cohen's D: 0.908), with a large effect size ${ }^{(22)}$. Similar results were found in the second measurement of the perception of the mother's general health, measured using the SF-12 scale ( $p: 0.001)$ CG (Mean: 79.91 SD: 15.55) vs EG (Mean: 89.44, SD: 12.689) (Cohen's D: 0.736), with a large effect size ${ }^{(22)}$.

In the physiological adaptive mode: nutrition, the results show a favorable effect of the therapy on neonatal growth and sucking ability. With regard to weight, height and growth in head circumference in newborns, the MannWhitney $U$ test showed statistically significant differences. Weight gain at the first measurement point ( $p: 0.009)$ CG (Mean: 4.34 grams, SD: 16.95) vs EG (Mean: 12.69 grams, SD: 13.41) (Cohen's D: 0.54) showed a large effect size ${ }^{(22)}$. In the second measurement $(p: 0.000)$ GC (Mean: 29.10 grams, SD: 8.01) vs GE (Mean: 33.29 grams, SD: 7.81) (Cohen's D: 0.53), it was observed a large effect size ${ }^{(22)}$. Height and head circumference showed differences in the second measurement. Height measurement ( $p$ : 0.025) CG (Mean: $0.084 \mathrm{~cm}, \mathrm{SD}$ : 0.05) vs EG (Mean: $0.109 \mathrm{~cm}, \mathrm{SD}:$ 0.05) (Cohen's D: 0.4 ) showed a small effect size ${ }^{(22)}$. Head circumference measurement ( $p$ : 0.041) CG (Mean: $0.0865 \mathrm{~cm}, \mathrm{SD}$ : 0.029 ) vs EG (Mean: $0.1007 \mathrm{~cm}, \mathrm{SD}: 0.31$ ) (Cohen's D: 0.78 ) showed a large effect size ${ }^{(22)}$.

In the interdependence adaptive mode: Appropriate Affection and Proper Development, the statistical tests show that the ATVV stimulation prevents PPD, measured by the Edinburgh Scale, Mann-Whitney $U$ test ( $p$ : 0.000) CG (Mean: 6.91, SD: 4.11) vs EG (Mean: 4.31, SD: 2.83) (Cohen's D: $0.632)$, with a medium effect size ${ }^{(22)}$. Its also improves the synchronism in the mother-child interaction and decreases the risk of stress related to maternal role, measured by the BaM-13 scale, Mann-Whitney $U$ test (p: 0.001) CG (Mean: 9.6, SD: 5.29) vs EG (Mean: 6.19, SD: 4.12) (Cohen's D: 0.662), with a medium effect size ${ }^{(22)}$. These results were amply described in other research articles.
The variables were submitted to a logistic regression analysis, including the different variables considered as important in the literature, which were accessed in the statistical program by pressing the enter key. These include: mode of birth, monthly income, level of education, sex, weight and gestational age of the newborn, complications during pregnancy, physical pain in the postpartum period, perception of the mother's health, her physical function and performance of her role, emotional state of the mother, amount of night sleep of the mother and child, synchronism in their interrelation, age and feeding of the newborn. Several models were analyzed including the following aspects: 1) the correct classification percentage of the model, 2) the Chi square test, 3 ) the decrease in the deviation ( -2 times the Likelihood Logarithm). Finally, a regression model to explain the risk of stress related to maternal role measured in the second week of postnatal life was proposed with the following properties: sensitivity of $69.8 \%$, specificity of $92.2 \%$; total adjustment percentage of $83.2 \%$; Chi square: 45.822 ( $p$ : 0.000), which ensures that independent variables can explain the response variable; -2 times the Likelihood Logarithm: 98.363. These characteristics explain $47.1 \%$ (0.471: Nagelkerke's R squared) of variance of the risk of maternal stress. These data are shown in Table 3.

These characteristics allow us to select the previous regression model and qualify it as a good model to explain the risk of stress related to maternal role using 3 independent variables. It is concluded that, the older the mother, the lower the risk of stress related to maternal role (OR: 0.890, p: 0.034). In addition, the use of a nursing bottle to feed the newborn increases the risk (OR: 4.566, $p$ : 0.026), and high scores on the scale of postnatal depression increases the risk of stress related to maternal role (OR: $1.556, p:$ 0.000). Another remarkable finding is that belonging to the experimental or control group has no influence on the response variable. This result is consistent with the tests to estimate the differences between groups, used for measurements during the first 2 weeks of postnatal life.

Based on Roy's adaptation model, it is confirmed that the mother-child dyad is an Integrated System, which cannot be interpreted in isolation, since the conditions that affect one also affect the other, so its adaptation must be understood jointly. 


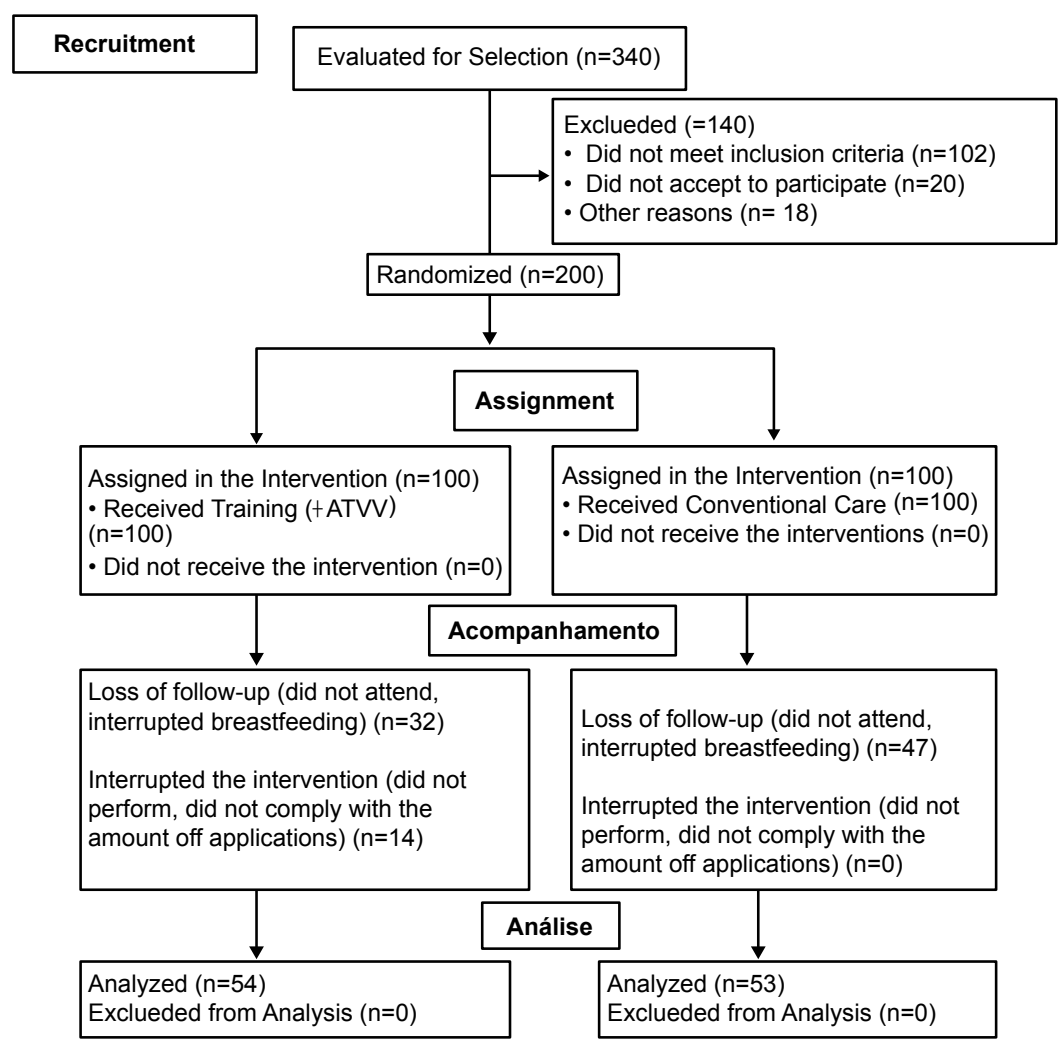

*CONSORT: Consolidated Standards of Reporting Trials; †ATVV: Auditory, Tactile, Visual and Vestibular Stimulation

Figure 2 - Flow chart of participants in accordance with the statement of the Consolidated Standards of Reporting Trials (CONSORT*). Bogotá D.C, Colombia, 2016

Table 1 - Socioeconomic characteristics of mothers. Bogotá D.C., Colombia, 2016

\begin{tabular}{|c|c|c|c|c|c|}
\hline \multicolumn{3}{|c|}{ Control Group $(n=53)$} & \multicolumn{2}{|c|}{ Experimental Group $(n=54)$} & \multirow{2}{*}{$\begin{array}{c}\mathrm{T}^{\dagger} \\
(<0.05)\end{array}$} \\
\hline Variables & Mean & SD* & Mean & SD* $^{*}$ & \\
\hline Age & 25.2 & 6.2 & 24.3 & 4.2 & $(0.9)$ \\
\hline Monthly income & $1062115,4 \mathrm{COP}^{\ddagger}$ & $792566,8 \mathrm{COP}^{\ddagger}$ & $\begin{array}{c}1055090,9 \\
\mathrm{COP}^{\ddagger}\end{array}$ & $683874,8 \mathrm{COP}^{\ddagger}$ & $(0.4)$ \\
\hline Years of study & 12.9 & 2.5 & 13.3 & 2.1 & $(0.3)$ \\
\hline Length of Labor in Hours & 7.5 & 4.9 & 8.1 & 5.5 & $(0.6)$ \\
\hline
\end{tabular}

*SD = Standard Deviation; ${ }^{\dagger} \mathrm{T}=$ Mann-Whitney $\mathrm{U}$ test; ${ }^{\ddagger} \mathrm{COP}=$ Colombian Peso

Table 2 - Characteristics of newborns at birth. Bogotá D.C, Colombia, 2016

\begin{tabular}{|c|c|c|c|c|c|c|c|c|c|}
\hline \multicolumn{5}{|c|}{ Control Group n=53 } & \multicolumn{4}{|c|}{ Experimental Group n=54 } & \multirow{2}{*}{$\begin{array}{c}T^{\S} \\
<0.05\end{array}$} \\
\hline Variables & $\operatorname{Min}^{*}$ & Máx ${ }^{\dagger}$ & Mean & SD $\ddagger$ & Min* & Máx ${ }^{\dagger}$ & Mean & SD $\ddagger$ & \\
\hline Weight & 2560 & 4100 & 3152.7 & 361.4 & 2550 & 4080 & 3077.3 & 362.9 & $(0.3)$ \\
\hline Height & 47 & 55 & 50.7 & 1.8 & 47 & 53 & 50.3 & 1.4 & $(0.2)$ \\
\hline Head Circumference & 31 & 36 & 33.9 & 1.2 & 31.5 & 36 & 33.7 & 1.1 & $(0.4)$ \\
\hline Gestational age & 37 & 40 & 39.0 & 0.9 & 37 & 40 & 38.9 & 1.0 & $(0.9)$ \\
\hline Apgar at minute & 7 & 8 & & & 6 & 9 & & & $(0.09)$ \\
\hline Apgar at 5 minutes & 8 & 9 & & & 8 & 9 & & & $(0.09)$ \\
\hline
\end{tabular}

${ }^{*}$ Min = Minimum; ${ }^{+}$Max = Maximum; ${ }^{\ddagger} \mathrm{SD}=$ Standard Deviation; ${ }^{\circledR} \mathrm{T}=$ Mann-Whitney $\mathrm{U}$ test

Table 3 - Logistic regression model: stress related to maternal role in the $2^{\text {nd }}$ week. Bogotá D.C, Colombia, 2016

\begin{tabular}{|c|c|c|c|c|c|c|}
\hline \multicolumn{7}{|c|}{ Variables in the equation } \\
\hline $1^{\text {st }}$ Stage & B & $\begin{array}{c}\text { Standard } \\
\text { error }\end{array}$ & Wald & df & Sig. & $\operatorname{Exp}(B)$ \\
\hline Age of the mother & -0.116 & 0.055 & 4.505 & 1 & $(0.034)$ & 0.890 \\
\hline Type of feeding of the $\mathrm{RN}^{*}$ & 1.519 & 0.681 & 4.970 & 1 & $(0.026)$ & 4.566 \\
\hline PPD Scale (EPDS) ${ }^{\dagger}$ & 0.442 & 0.097 & 20.819 & 1 & $(0.000)$ & 1.556 \\
\hline Constant & -0.754 & 1.299 & 0.337 & 1 & $(0.562)$ & 0.471 \\
\hline
\end{tabular}

*Type of feeding of the NB = (0: Breastfeeding), (1: Mixed Feeding); ${ }^{+P P D}$ Scale (EPDS) = Edinburgh Scale 


\section{Discussion}

Despite the efforts of the researchers to carry out the follow-up of the mother-child dyads, there was a large loss of participants during this study. Living conditions that are typical in a country in conflict, such as internal displacement and insecurity, which makes it difficult to use other forms of follow-up, such as home follow-up. It is possible that these findings are due to the pain and mobility limitations of mothers in using public transport during this short period, as well as the cultural practice of moving to the maternal home for quarantine care, even when it is necessary to move to another city. In view of the results in the Physiological Adaptive Mode, this study supports the results previously found by authors from Korea(17) and Colombia(23) on the positive effect of the stimulation therapy on growth; as well as the favorable effect on the sucking ability mentioned in previous studies conducted in the United States ${ }^{(16,24-25)}$. Regarding the findings of this study on activity and exercise, no other authors who have applied this therapy before in the infantile and maternal sleep and in the general health of the woman in the puerperium were found. The results of the effect of this therapy in the Interdependence Adaptive Mode coincide with those found in studies conducted with mothers of extremely premature newborns, comparing with the results of $\mathrm{PPD}^{(15)}$. No study measuring the effect of ATVV therapy on stress related to maternal role was found.

On the regression model, it is important to analyze the influence of the type of feeding, since the sucking ability is the expression of the organization of newborn behavior. The establishment of breastfeeding depends on the vigorous and continuous sucking of the newborn from its birth, which can positively or negatively affect the production of breast milk(26-27), and indirectly affect the amount of milk that the newborn can to suck. When this process does not take place in a harmonious way, mothers make use of bottle feeding and breast milk substitutes ${ }^{(28)}$. Therefore, the evidence shows that there is a negative relationship between bottle-feeding and the sucking ability of the newborn and the establishment of breastfeeding, which delays the organization of behavior. In this way, having a slower organization of behavior leads to a greater maternal emotional imbalance during the first days of life. These findings are consistent with those of previous studies that point out the relationship between breastfeeding difficulties and an increased risk of PPD(28), but the evidence in this regard is still inconclusive.

This study recognizes among its limitations the lack of budget and the large loss of participants during follow-up, which caused the extension of the data collection period to attenuate it. Additionally, no strategies were available to control the application of home therapy doses. Other studies have used tools such as diaries or written records of participants, and no data on the instrumentation used during vaginal deliveries were obtained, which may have some influence on aspects such as the general health of mothers in the postpartum period.

\section{Conclusions}

The multimodal stimulation therapy ATVV applied by mothers to their healthy newborns, at home, generates important benefits especially in contexts of vulnerability and resource shortages. The results show a positive effect on the sooner neurobehavioral organization, demonstrated in the sucking ability of the newborn, as well as in the favorable evolution of anthropometric measures (weight, height and head circumference). These findings are relevant to the professional nursing practice, since its effects allow the application of a strategy with low cost and with no known risks for a wide range nutritional risk conditions for the newborn. Another important aspect is the emotional adjustment of the mother, since the therapy is shown to be an effective tool in the prevention of emotional damages such as the stress related to maternal role and PPD. The latter is considered a major problem for the women's health worldwide. Therefore, this intervention should be continued and promoted in order to facilitate the adaptation of the mother-child dyad to postnatal life.

The application of early stimulation therapies, such as ATVV therapy in teaching for healthy children, which should be provided by nurses to the mothers, allows the practice of evidence-based nursing and enriches the tools for health promotion and disease prevention.

\section{Acknowledgments}

The authors thank each of the mothers of newborns who agreed to participate in this study, because this study could not have been carried out without their 
provision. In the same way, they thank the Hospital staff, who showed a positive attitude and offered their collaboration during the data collection stage.

\section{References}

1. Császár-Nagy N, Bókkon I. Mother-newborn separation at birth in hospitals: A possible risk for neurodevelopmental disorders? Neurosci Biobehav Rev. [Internet]. 2018[cited Feb 25, 2019] 84(April 2017):337-51. Available from: http://doi.org/10.1016/j. neubiorev.2017.08.013

2. Leclère $C$, Viaux $S$, Avril $M$, Achard $C$, Chetouani $M$, Missonnier $S$, Cohen D. Why synchrony matters during mother-child interactions: A systematic review. PLoS ONE. [Internet]; 2014 [cited Feb 25, 2019] 9(12):135. Available from: http://doi.org/10.1371/journal. pone.0113571

3. Van der Woude DA, Pijnenborg JMA, de Vries J. Health status and quality of life in postpartum women: a systematic review of associated factors. Eur J Obstet Gynecol Reprod Biol. [Internet]; 2015[cited June 5, 2018];185:45-52. Available from: http://linkinghub. elsevier.com/retrieve/pii/S0301211514006320

4. Tamilia E, Taffoni F, Formica D, Ricci L, Schena E, Keller $F$, et al. Technological solutions and main indices for the assessment of newborns' Nutritive Sucking: A review. Sensors. (Switzerland) [Internet]. 2014 [cited Feb 25, 2019]14(1):634-58. Available from: http://doi. org/10.3390/s140100634

5. White-Traut R, Rankin KM, Medoff -Cooper B, Liu L, Shapiro N, Lucas R. Evaluating sucking maturation using two pressure thresholds. Early Hum Dev. [Internet]. 2013[cited June 5, 2018];89(10):833-7. Available from: https://www.ncbi.nlm.nih.gov/pubmed/23972294

6. Fonseca $M J$, Severo $M$, Barros $H$, Santos AC. Determinants of weight changes during the first 96 hours of life in full-term newborns. Birth. [Internet]. 2014[cited June 5, 2018];41(June):160-8. Available from: https://doi.org/10.1111/birt.12087

7. Mayock DE, Gleason CA. Neonatal Pain and Stress : Assessment and Management. In: Gleason CA, Juul SE. Avery's Diseases of the Newborn [Internet]. 2017. [cited Feb 25, 2019] p. 429-44. Available from: http://dx.doi. org/10.1016/B978-1-4377-0134-0.10035-6

8. Relland LM, Gehred A, Maitre NL. Behavioral and Physiological Signs for Pain Assessment in Preterm and Term Neonates During a Nociception-Specific Response: A Systematic Review. Pediatr Neurol. [Internet]. 2018 [cited Feb 25, 2019];90 (2019): 13-23. Available from: https://doi.org/10.1016/j.pediatrneurol.2018.10.001

9. Müller EB, Mota M de F. Educative practice with nurses, with a view to humanized care for the newborn in the
Obstetric Center. Texto Contexto Enferm. [Internet]. 2014[cited June 5, 2018];23(3):782-90. Available from: http://dx.doi.org/10.1590/0104-07072014002250013 10. Charandabi SM-A, Mirghafourvand M, Sanaati F. The Effect of Life Style Based Education on the Fathers' Anxiety and Depression During Pregnancy and Postpartum Periods: A Randomized Controlled Trial. Commun Ment Health J. [Internet]. 2017[cited Feb 25, 2019]; 53:483-9. Available from: http://link.springer. com/10.1007/s10597-017-0103-1

11. Canestraro L, Favero L, Carraro TE, Wall ML. The primiparous mother at home and nursing: grounded theory. Online Braz ] Nurs. [Internet]. 2015[cited June 5, 2018];11(3):1-12. Available from: https:// www.objnursing.uff.br/index.php/nursing/article/ viewFile/3852/pdf

12. Bergmann RL, Bergmann $\mathrm{KE}$, von Weizsäcker $\mathrm{K}$, Berns M, Henrich W, Dudenhausen JW. Breastfeeding is natural but not always easy: intervention for common medical problems of breastfeeding mothers - a review of the scientific evidence. J Perinat Med. [Internet]. 2014 Jan [cited Feb 25, 2019];42(1):9-18. Available from: http://www.ncbi.nlm.nih.gov/pubmed/24057589

13. Camargo-Figuera FA, Barros AJ, Santos IS, Matijasevich A, Barros FC. Early life determinants of low IQ at age 6 in children from the 2004 Pelotas Birth Cohort: a predictive approach. BMC Pediatr. [Internet]. 2014[cited June 5, 2018];14(1):1-12. Available from: http://www.biomedcentral.com/1471-2431/14/308

14. Jolly $K$, Ingram L, Khan K, Freemantle N, MacArthur C. Systematic review of peer support for breastfeeding continuation: metaregression analysis of the effect of setting, intensity, and timing. Natl Inst Health Res. [Internet].2014[cited Feb 25, 2019];1-3. Available from: http://www.bmj.com/content/344/bmj.d8287 15. Holditch-Davis D, White-Traut RC, Levy J a, Shea TMO, Geraldo V, David RJ. Infant behavior and development full length article maternally administered interventions for preterm infants in the NICU: Effects on maternal psychological distress and mother - infant relationship. Infant Behav Dev. [Internet]; 2014[cited June 5, 2018];37(4):695-710. Available from: http:// dx.doi.org/10.1016/j.infbeh.2014.08.005

16. Medoff-Cooper B, Rankin KM, Zhuoying L, Liu L, White-Traut R. Multi-sensory intervention for preterm infants improves sucking organization. Adv Neonatal Care. [Internet]. 2015[cited June 5, 2018];15(2): 142-9. Available from: http://dx.doi.org10.1097/ ANC.0000000000000166

17. Kim TI, Shin YH, White-Traut RC. Multisensory intervention improves physical growth and illness rates in Korean orphaned newborn infants. Res Nurs Health. 
[Internet]. 2003[cited June 5, 2018];26:424-33. Available from: https://doi.org/10.1002/nur.10105

18. Thomas KA, Spieker S. Sleep, Depression, and Fatigue in Late Postpartum. MCN Am J Matern Child Nurs. [Internet].2016 [cited Feb 25, 2019];41(2): 104-9. Available from: https://dx.doi.org.10.1097/ NMC. 0000000000000213

19. Burns K, Cunningham N, White-Traut RC, Silvestri J, Nelson M. Infant stimulation: modification of an intervention based on physiological and behavioral cues. J Obs Gynecol Neonat Nurs. [Internet]. 1994 [cited June 5, 2018];23:581-9. Available from: https://www.ncbi. nlm.nih.gov/pubmed/7996309

20. Roy SC. The Roy Adaptation Model. 3.rd.ed. New Jersey: Pearson Education; 2009. 553 p.

21. León-Sánchez L, Linares-Guerra E. The binary logistic regression as an instrument for the prediction of immunological deterioration from nutritional indicators in people. Rev Investig Operacional. [Internet]. 2014[cited June 5, 2018];35(1):35-48. Available from: https:// www.invoperacional.uh.cu/index.php/InvOp/article/ download/430/394

22. Cardenas Castro M, Arancibia Martini H. Statistical power and effect size calculating in G*Power: complementary analysis of statistical significance test and its application in Psicology. Salud Soc. [Internet]. 2014[cited Feb 28, 2019];5(2):210-24. Available from: https://doi.org/10.22199/S07187475.2014.0002.00006 23. Villamizar B, Duran MM. Roy's adaptation model in a controlled clinical trial. Av Enferm. [Internet]. 2012[cited June 5, 2018];30(2):97-107. Available from: http:// www.scielo.org.co/pdf/aven/v30n2/v30n2a09.pdf 24. White-Traut R. Sucking Organization Following an Infant-Directed Developmental Intervention. J Obstet Gynecol Neonatal Nurs. [Internet]. 2013[cited June 5, 2018];42:S88. Available from: http://linkinghub. elsevier.com/retrieve/pii/S0884217515313988

25. White-Traut R, Rankin KM, Yoder J, Liu L, Vasa R, Geraldo $V$, et al. Influence of H-HOPE intervention for premature infants on growth, feeding progression, and length of stay during initial hospitalization. J Perinatol. [Internet]. 2015[cited June 5, 2018];35(8):636-41. Available from: https://doi.org/10.1038/jp.2015.11

26. Bolio-Molina LA. Lactogenesis in the first five days of puerperium and lactation. Rev Mex Pediatr. [Internet]. 2013[citado June 5, 2018];80(1):10-4. Available from: http://www.medigraphic.com/pdfs/pediat/sp2013/sp131c.pdf

27. Bergmann RL, Bergmann KE, von Weizsäcker $\mathrm{K}$, Berns M, Henrich W, Dudenhausen JW. Breastfeeding is natural but not always easy: intervention for common medical problems of breastfeeding mothers - a review of the scientific evidence. J Perinat Med. [Internet]. 2014 Jan[cited June 5, 2018];42(1):9-18. Available from: http://www.ncbi.nlm.nih.gov/pubmed/24057589

28. Pope CJ, Mazmanian D, Bédard M, Sharma V. Breastfeeding and postpartum depression: Assessing the influence of breastfeeding intention and other risk factors. ] Affect Disorders. [Internet]. 2016[cited June 5, 2018];200:45-50. Available from: https://doi. org/10.1016/j.jad.2016.04.014
Received: Dec $13^{\text {th }} 2018$

Accepted: Apr $3^{\text {rd }} 2019$
Copyright ( $) 2019$ Revista Latino-Americana de Enfermagem This is an Open Access article distributed under the terms of the Creative Commons (CC BY).

This license lets others distribute, remix, tweak, and build upon your work, even commercially, as long as they credit you for the original creation. This is the most accommodating of licenses offered. Recommended for maximum dissemination and use of licensed materials. 


\section{Erratum}

In page 3 , Where was written:

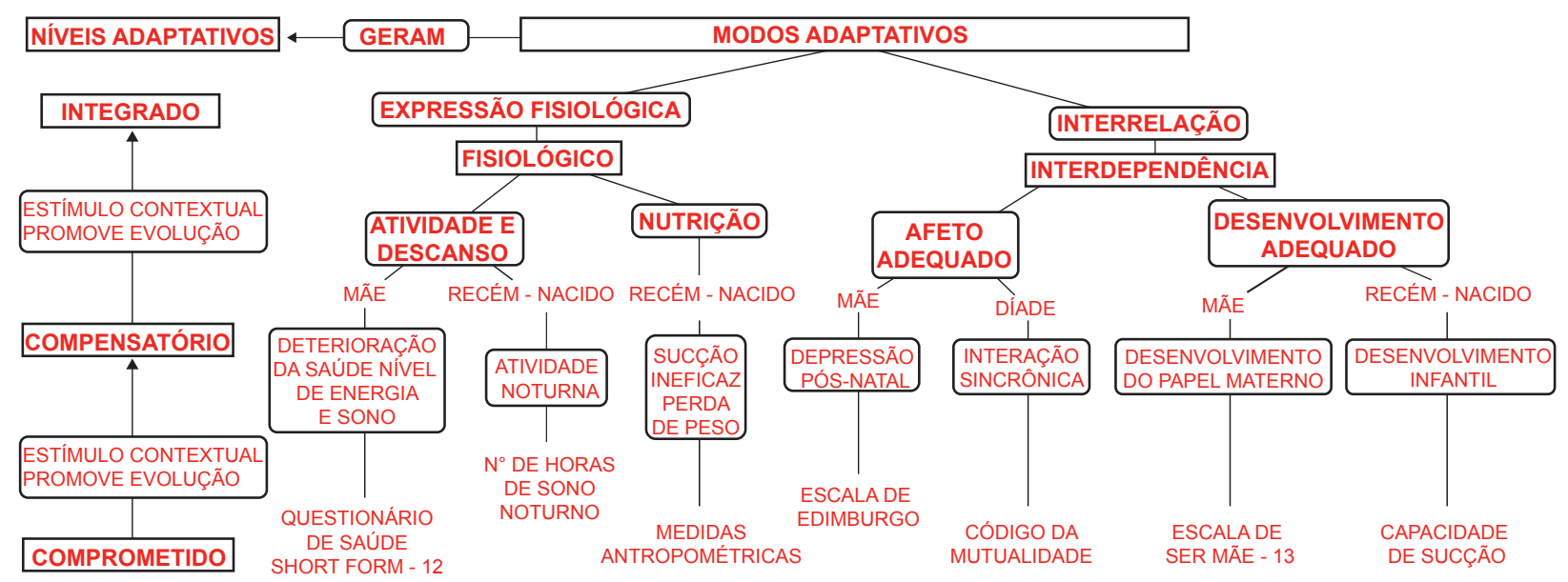

Figure 1 - Roy's Adaptation Model and Outcome Variables. Bogotá D.C, Colombia, 2016

Now read:

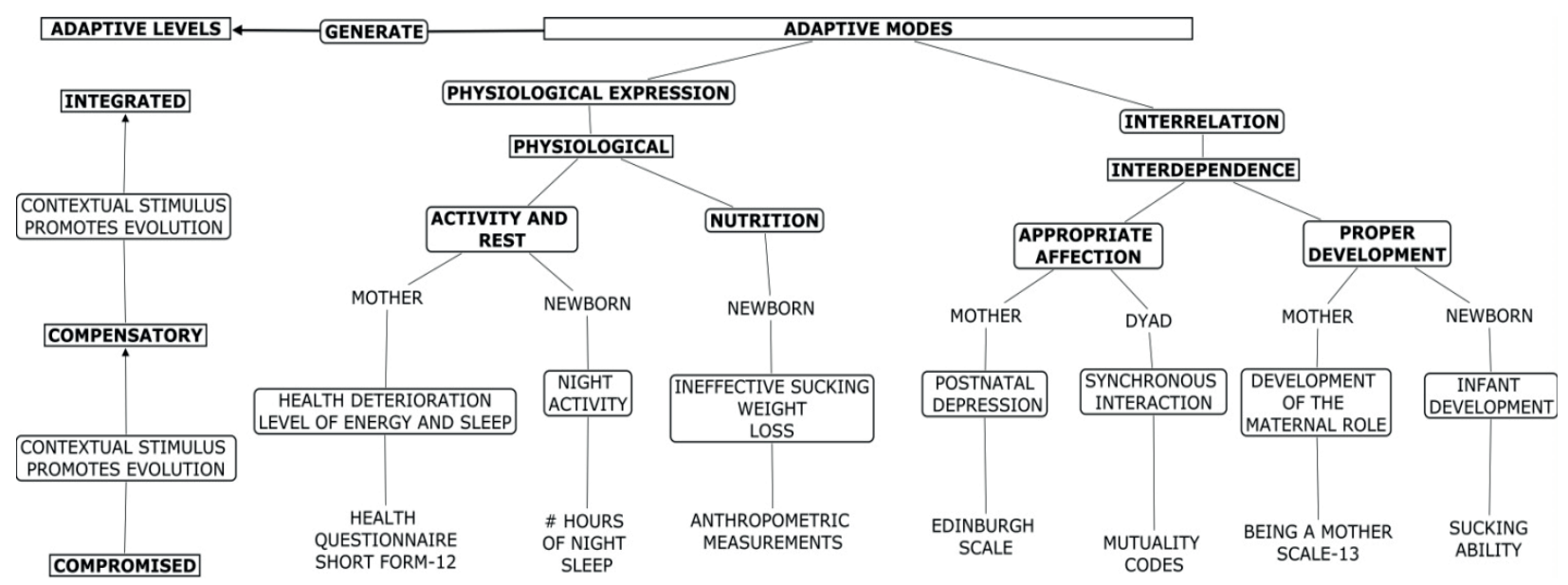

Figure 1 - Roy's Adaptation Model and Outcome Variables. Bogotá D.C, Colombia, 2016

\section{Rev. Latino-Am. Enfermagem}

2019;27:e3244 